\title{
REGULAR MAPS WITH SMALL PARAMETERS
}

\section{MARSTON CONDER}

(Received 15 July 1993)

\author{
Communicated by L. G. Kovács
}

\begin{abstract}
Regular maps of type $\{p, q\}_{r}$ and the associated groups $G^{p, q, r}$ are considered for small values of $\grave{p}, q$ and $r$. In particular, it is shown that the groups $G^{4,6,6}$ and $G^{5,5,6}$ are Abelian-by-finite, and there are infinitely many regular maps of each of the types $\{4,6\}_{6},\{5,5\}_{6},\{5,6\}_{6}$ and $\{6,6\}_{6}$.

1991 Mathematics subject classification (Amer. Math. Soc.): primary 05C25; secondary 20F05, 57M15.
\end{abstract}

\section{Introduction}

A map $M$ is a 2-cell embedding of a connected graph (or multigraph) into some closed surface $S$, dividing $S$ into simply-connected regions called faces of the map. We use $V(M), E(M)$ and $F(M)$ to denote the sets of vertices, edges and faces of $M$, respectively. An automorphism of a map $M$ is any automorphism of the underlying graph (or multigraph) which preserves also the faces of the embedding - or, what is essentially equivalent, a homeomorphism of the surface $S$ preserving $V(M)$ and $E(M)$ and necessarily also $F(M)$. As usual, these automorphisms form a group under composition, called the automorphism group of the map $M$, and denoted by Aut $M$.

If Aut $M$ contains an automorphism $A$ cyclically permuting the edges bounding a face $f$ (in single steps around $f$ ), and an automorphism $B$ cyclically permuting the edges incident to a vertex $v$ of this face (in single steps around $v$ ), then $M$ is called a rotary map; see [6]. In this case, by connectedness Aut $M$ acts transitively on the arcs (ordered edges) of $M$. Also by conjugation, it follows that every face is bounded by the same number of edges, say $p$, and every vertex is incident with the same number of edges, say $q$, and the map is then said to be of type $\{p, q\}$. Counting the number of arcs of $M$ in three different ways gives the well known

(C) 1994 Australian Mathematical Society 0263-6115/94\$A2.00+0.00 
identity $q|V(M)|=2|E(M)|=p|F(M)|$, and the Euler characteristic of the surface $S$ may be calculated using the usual formula $\chi=|V(M)|-|E(M)|+|F(M)|$; see $[4, \S 8.1]$. The automorphisms $A$ and $B$ have orders $p$ and $q$ respectively, and their product $A B$ 'flips' one of the edges incident to $v$ in $f$ (while interchanging $f$ with the other face to which this edge belongs), and so has order 2 .

Following [6], we define a regular map to be a rotary map which admits also a reflection, flipping an edge $e$ but fixing the two faces to which $e$ belongs. Note that in [4] the term 'regular' is used for maps which are rotary (according to the current definition), and 'reflexible' is used for maps we now call regular.

Suppose $M$ is a regular map of type $\{p, q\}$, with automorphisms $A$ and $B$ defined (for a given face $f$ and incident vertex $v$ ) as above. Also let $e$ be the edge incident to $v$ in $f$ that is flipped by $A B$, so that $(v, e, f)$ is a mutually incident vertex-edge-face triple, or $f$ lag of $M$. If the automorphism $R_{1}$ is chosen to flip this edge $e$ while fixing $f$, then $R_{2}=R_{1} A$ and $R_{3}=R_{1} A B$ are also reflections:

$R_{1}$ fixes $e$ and $f$ (but interchanges $v$ with one of its neighbours on $f$ ), while $R_{2}$ fixes $v$ and $f$ (but interchanges $e$ with one of the other edges incident to $v$ ), and

$R_{3}$ fixes $v$ and $e$ (but interchanges $f$ with the other face containing $e$ ).

In particular, each of $R_{1}, R_{2}$ and $R_{3}$ has order 2, while $R_{1} R_{2}(=A), R_{2} R_{3}(=B)$ and $R_{1} R_{3}(=A B)$ have orders $p, q$ and 2 respectively. Moreover, $R_{1}, R_{2}$ and $R_{3}$ generate the group Aut $M$, which is transitive (indeed regular) on the set of all flags $(v, e, f)$ of $M$, every automorphism being uniquely determined by its effect on any flag. Also, as every edge lies in four different flags, it follows easily that $|A u t M|=4|E(M)|$. The composite automorphism $R_{1} R_{2} R_{3}$ acts like a glide reflection, moving the edge $e$ along a Petrie path: a 'zig-zag' circuit in which every two but no three consecutive edges share a common incident vertex-face pair. In particular, if this path is of length $r$ then $M$ is said to be of type $\{p, q\}_{r}$, and we may now call $p, q$ and $r$ the parameters of the regular map $M$.

In the latter case, the three reflections $R_{1}, R_{2}$ and $R_{3}$ satisfy the relations $R_{1}^{2}=$ $R_{2}^{2}=R_{3}^{2}=\left(R_{1} R_{2}\right)^{p}=\left(R_{2} R_{3}\right)^{q}=\left(R_{1} R_{3}\right)^{2}=\left(R_{1} R_{2} R_{3}\right)^{r}=1$. The map $M$ is orientable or non-orientable according as to whether the subgroup generated by $R_{1} R_{2}$ and $R_{2} R_{3}$ has index 2 or 1 in Aut $M$; in particular, when $r$ is odd, this index has to be 1 and so $M$ is non-orientable. In the orientable case, the genus $g$ of the surface $S$ (and hence of the map $M)$ is given by $2-2 g=\chi=\mid$ Aut $M \mid(1 / 2 q-1 / 4+1 / 2 p)$.

Also these relations are defining relations for the abstract group $G^{p, q, r}$, as discussed in [4]. Taking $A=R_{1} R_{2}, B=R_{2} R_{3}$ and $C=\left(R_{1} R_{2} R_{3}\right)^{-1}=R_{3} R_{2} R_{1}$ as alternative generators, we have

$$
G^{p, q, r}=\left\langle A, B, C \mid A^{p}=B^{q}=C^{r}=(A B)^{2}=(B C)^{2}=(C A)^{2}=(A B C)^{2}=1\right\rangle,
$$


or equivalently, putting $x=A B, y=A$ and $t=B C$,

$$
G^{p, q, r}=\left\langle x, y, t \mid x^{2}=y^{p}=(x y)^{q}=t^{2}=(x t)^{2}=(y t)^{2}=(x y t)^{r}=1\right\rangle .
$$

Given the symmetry of these presentations, it is not difficult to show that $G^{p, q, r}$ is isomorphic to $G^{k, l, m}$ for any permutation $(k, l, m)$ of $(p, q, r)$, so this group may be associated with regular maps of up to six types: $\{p, q\}_{r},\{p, r\}_{q},\{q, p\}_{r},\{q, r\}_{p}$, $\{r, p\}_{q}$ and $\{r, q\}_{p}$. (See [4] or [6] for further details on this.)

Clearly the automorphism group of every regular map of type $\{p, q\}_{r}$ is a homomorphic image of $G^{p, q, r}$. On the other hand, any non-degenerate homomorphism (preserving the orders of the generators $A, B$ and $C$ ) of $G^{p, q, r}$ to a finite group $G$ gives rise to a regular map of type $\{p, q\}_{r}$, as follows:

First take the universal tessellation $\{p, q\}$ of the sphere, Euclidean plane or hyperbolic plane (depending on the value of $1 / p+1 / q+1 / r$ ), and identify pairs of vertices at distance $r$ along a Petrie path, as in $[4, \S 8.6])$. At this stage we have a map with $G^{p, q, r}$ as its automorphism group. Now if $K$ is the kernel of the homomorphism from $G^{p, q, r}$ to $G$, simply identify those pairs of vertices in the same orbit of the normal subgroup $K$. The resulting map is of type $\{p, q\}_{r}$, with $G$ as its automorphism group.

(In particular, for some flag $(v, e, f)$ of this map, the images of $B$ and $A C$ generate the stabilizer of $v$, while the images of $A B$ and $C A$ generate the stabilizer of $e$, and the images of $A$ and $B C$ generate the stabilizer of $f$; see earlier.)

In this paper, we consider regular maps with small parameters, and in particular those with $p, q$ and $r$ all at most 6 .

Many such maps were found by Coxeter in the course of his investigation [3] into which of the groups $G^{p, q, r}$ are finite; see also [4, Table 8]. Some of this information will be summarized in Section 2. Unfortunately the investigation begun in [3] has not been completed: for example it is still not known whether $G^{3,7,19}$ is finite. On the other hand, for some $p, q, r$, the group $G^{p, q, r}$ is known not only to be infinite, but also to have all but finitely many alternating and symmetric groups as quotients, so that every finite group is a subgroup of some factor group of $G^{p, q, r}$; for example, this is true of $G^{6,6,6}$ (see [2]). The infinite cases of $G^{p, q, r}$ (and associated regular maps) with $p, q$ and $r$ all at most 6 are discussed in Section 3, and some open questions are given in Section 4.

\section{Finite cases}

The following list describes all the cases where the group $G^{p, q, r}$ is finite, for $2 \leq p \leq q \leq r \leq 6$. Recall that each such group gives rise to up to six different regular maps, of types $\{p, q\}_{r},\{p, r\}_{q},\{q, p\}_{r},\{q, r\}_{p},\{r, p\}_{q}$ and $\{r, q\}_{p}$. Also note that when the order of $G^{p, q, r}$ is 1,2 or 4 , the group is trivial, $C_{2}$ or $C_{2} \times C_{2}$ respectively, 
and in these cases the associated maps are all trivial. In all other cases the numbers of vertices, edges and faces of the associated maps may be calculated by the formulae given in the Introduction.

\begin{tabular}{|c|c|c|c|c|}
\hline$p$ & $q$ & $r$ & $\left|G^{p, q, r}\right|$ & $G^{p, q, r}$ \\
\hline 2 & 2 & 2 & 8 & $C_{2} \times C_{2} \times C_{2}$ \\
\hline 2 & 2 & 3 & 4 & \\
\hline 2 & 2 & 4 & 8 & $C_{2} \times C_{2} \times C_{2}$ \\
\hline 2 & 2 & 5 & 4 & \\
\hline 2 & 2 & 6 & 8 & $C_{2} \times C_{2} \times C_{2}$ \\
\hline 2 & 3 & 3 & 6 & $D_{3}$ \\
\hline 2 & 3 & 4 & 4 & \\
\hline 2 & 3 & 5 & 2 & \\
\hline 2 & 3 & 6 & 12 & $D_{6}$ \\
\hline 2 & 4 & 4 & 16 & $D_{4} \times C_{2}$ \\
\hline 2 & 4 & 5 & 4 & \\
\hline 2 & 4 & 6 & 8 & $C_{2} \times C_{2} \times C_{2}$ \\
\hline 2 & 5 & 5 & 10 & $D_{5}$ \\
\hline 2 & 5 & 6 & 4 & \\
\hline 2 & 6 & 6 & 24 & $D_{6} \times C_{2}$ \\
\hline 3 & 3 & 3 & 1 & \\
\hline 3 & 3 & 4 & 24 & $S_{4}$ \\
\hline 3 & 3 & 5 & 1 & \\
\hline 3 & 3 & 6 & 6 & $D_{3}$ \\
\hline 3 & 4 & 4 & 4 & \\
\hline 3 & 4 & 5 & 2 & \\
\hline 3 & 4 & 6 & 48 & $S_{4} \times C_{2}$ \\
\hline 3 & 5 & 5 & 60 & $A_{5}$ \\
\hline 3 & 5 & 6 & 2 & \\
\hline 3 & 6 & 6 & 108 & $\left(C_{3} \times C_{3}\right) \cdot D_{6}$ \\
\hline 4 & 4 & 4 & 64 & $\left(C_{2} \times C_{2}\right) \cdot\left(D_{4} \times C_{2}\right)$ \\
\hline 4 & 4 & 5 & 4 & \\
\hline 4 & 4 & 6 & 144 & $\left(C_{3} \times C_{3}\right) \cdot\left(D_{4} \times C_{2}\right)$ \\
\hline 4 & 5 & 5 & 160 & $\left(C_{2} \times C_{2} \times C_{2} \times C_{2}\right) \cdot D_{5}$ \\
\hline 4 & 5 & 6 & 240 & $S_{5} \times C_{2}$ \\
\hline 5 & 5 & 5 & 660 & $L_{2}(11)$ \\
\hline
\end{tabular}

These and many of the other known finite cases (for larger $p, q$ and $r$ ) as given in [4] may be confirmed by coset enumeration using CAYLEY [1] or GAP [5].

\section{Infinite cases}

The only cases (for small $p, q$ and $r$ ) missing from the list in Section 1 are $G^{4,6,6}$, $G^{5,5,6}, G^{5,6,6}$ and $G^{6,6,6}$. These are dealt with one by one below. 
THEOREM 1. $G^{4,6,6}$ is an extension by $S_{4} \times C_{2}$ of a free Abelian group of rank 3 .

PROOF. Let $x, y$ and $t$ be generators for $G^{4,6,6}$ satisfying the relations $x^{2}=y^{4}=$ $(x y)^{6}=t^{2}=(x t)^{2}=(y t)^{2}=(x y t)^{6}=1$. As $(x y t)^{2}=x y t x y t=x y x t y t=$ $x y x y^{-1}$, we note the relation $\left(x y x y^{-1}\right)^{3}=1$ is satisfied also.

Now put $u=x y^{2} x y^{2} t, v=y^{-1} x y^{2} x y t$, and $w=x y^{-1} x y^{2} x y x t$. It is not difficult to show that these three elements and their inverses are conjugated among themselves by $x, y$ and $t$. First $x^{-1} u x=y^{2} x y^{2} t x=t y^{-2} x y^{-2} x=u^{-1}$, and similarly $y^{-1} u y=v$, $t^{-1} u t=u, x^{-1} v x=w, y^{-1} v y=u^{-1}, t^{-1} v t=v^{-1}, x^{-1} w x=v$, and $t^{-1} w t=w^{-1}$. Finally $y^{-1} w y=w^{-1}$, which may be seen as follows:

$$
\begin{array}{rlrl}
y^{-1} w y & =y^{-1} x y^{-1} x y^{2} x y x y^{-1} t & \\
& =y^{-1} x y^{-1} x y^{-1}\left(y^{-1} x y x y^{-1}\right) t & \\
& =y^{-1} x y^{-1} x y^{-1} x y^{-1} x y x y^{-1} x t & & \text { since }\left(x y x y^{-1}\right)^{3}=1 \\
& =x y x y^{2} x y^{-1} x t & & \text { since }(x y)^{5}=1 \\
& =t x y^{-1} x y^{2} x y x & & \\
& =w^{-1} . & &
\end{array}
$$

In particular, $u, v$ and $w$ generate a normal subgroup $K$ of the group $G=\langle x, y, t\rangle$.

But further, it follows that $u^{-1} v u=t y^{-2} x y^{-2} x v x y^{2} x y^{2} t=t y^{-2} x y^{-2} w y^{2} x y^{2} t$ $=t y^{-2} x w x y^{2} t=t y^{-2} v y^{2} t=t v^{-1} t=v$, so that $u$ and $v$ commute. Conjugating by $x$ and then by $y$, we find that $u$ and $w$ commute, as do $v$ and $w$, therefore $K$ is Abelian.

The factor group $G / K$ has presentation $\langle x, y, t| x^{2}=y^{4}=(x y)^{6}=t^{2}=(x t)^{2}=$ $\left.(y t)^{2}=(x y t)^{6}=x y^{2} x y^{2} t=1\right\rangle$, and by coset enumeration using CAYLEY [1] or GAP [5] this is easily shown to have order 48, being a direct product $S_{4} \times C_{2}$.

Finally, we show the group $G$ has a concrete representation of the form described in the statement of the theorem, by exhibiting the following transitive permutation representation on the integers:

$\begin{array}{ccccccc}x(8 k) & =8 k-4 & y(8 k) & =8 k+1 & t(8 k) & =8 k+1 \\ x(8 k+1) & =8 k-3 & y(8 k+1) & =8 k+2 & t(8 k+1) & =8 k \\ x(8 k+2) & =8 k+7 & y(8 k+2) & =8 k+3 & t(8 k+2) & =8 k+3 \\ x(8 k+3) & =8 k+6 & y(8 k+3) & =8 k & t(8 k+3) & =8 k+2 \\ x(8 k+4) & =8 k+8 & y(8 k+4) & =8 k+5 & t(8 k+4) & =8 k+5 \\ x(8 k+5) & =8 k+9 & y(8 k+5) & =8 k+6 & t(8 k+5) & =8 k+4 \\ x(8 k+6) & =8 k+3 & y(8 k+6) & =8 k+7 & t(8 k+6) & =8 k+7 \\ x(8 k+7) & =8 k+2 & y(8 k+7) & =8 k+4 & t(8 k+7) & =8 k+6 .\end{array}$

These permutations (defined for $k \in \mathbb{Z}$ ) satisfy the required relations, and generate a transitive but imprimitive group on $\mathbb{Z}$, with residue classes modulo 8 as blocks of imprimitivity. It is not difficult to show that the permutations induced by $u=x y^{2} x y^{2} t$, $v=y^{-1} x y^{2} x y t$, and $w=x y^{-1} x y^{2} x y x t$ generate a free Abelian group of rank 3 , and the rest follows. 
COROLLARY 1. For every positive integer $n$, there exists a regular map of type $\{4,6\}_{6}$ having $8 n^{3}$ vertices, $24 n^{3}$ edges and $12 n^{3}$ faces, on an orientable surface of genus $2 n^{3}+1$.

PROOF. Take the permutations given at the end of the above proof, modulo $8 n$; these generate a quotient of $G^{4,6,6}$ of order $96 n^{3}$, and the rest follows from material presented in the Introduction.

NOTE. Also when $n$ is even, the image of $u^{n / 2} v^{n / 2} w^{n / 2}$ generates a central subgroup of order 2, with corresponding factor group of order $48 n^{3}$ giving rise to a regular map of type $\{4,6\}_{6}$ having $4 n^{3}$ vertices, $12 n^{3}$ edges and $6 n^{3}$ faces, on an orientable surface of genus $n^{3}+1$. Similarly (when $n$ is even), the images of $u^{n / 2} v^{n / 2}$ and $u^{n / 2} w^{n / 2}$ generate a normal subgroup of order 4 , with corresponding factor group of order $24 n^{3}$ giving rise to a regular map of type $\{4,6\}_{6}$ having $2 n^{3}$ vertices, $6 n^{3}$ edges and $3 n^{3}$ faces, on an orientable surface of genus $n^{3} / 2+1$.

THEOREM 2. $G^{5,5,6}$ is an extension by $A_{5} \times C_{2}$ of a free Abelian group of rank 4 .

PROOF. Let $x, y$ and $t$ be generators for $G^{5,5,6}$ satisfying the relations $x^{2}=y^{5}=$ $(x y)^{5}=t^{2}=(x t)^{2}=(y t)^{2}=(x y t)^{6}=1$. As in the proof of Theorem 1, the relation $\left(x y x y^{-1}\right)^{3}=1$ is a consequence of these.

Now put $v_{0}=\left(x y^{2}\right)^{3}$, and $v_{i}=y^{-i} u_{0} y^{i}$ for $1 \leq i \leq 4$. These five elements generate a subgroup $K$ of $G=\langle x, y, t\rangle$ which is easily seen to be normalized by $y$ and by $t$; in fact $t^{-1} v_{i} t=v_{3-i}^{-1}$ for $0 \leq i \leq 3$, while $t^{-1} v_{4} t=v_{4}^{-1}$. Also $v_{0} v_{2} v_{4} v_{1} v_{3}=x y^{2}\left(x y^{4}\right)^{5} y^{-2} x=x y^{2} x\left(y^{4} x\right)^{5} x y^{-2} x=x y^{2} x(x y)^{-5} x y^{-2} x=1$, and so $K$ is generated by any four of the $v_{i}$. Next, it is easy to see that $x^{-1} v_{0} x=v_{3}$ and $x^{-1} v_{3} x=v_{0}$, and with a little more effort:

$$
\begin{aligned}
x^{-1} v_{1} x & =x y^{-1} x y^{2} x y^{2} x y^{-2} x & & \\
& =x y^{-1} x y^{2} x y^{-1} x y^{2} v_{0}^{-1} & & \\
& =y x y x y x y^{-2} x y^{-1} x y^{2} v_{0}^{-1} & & \text { since }(x y)^{5}=1 \\
& =y x y^{2} x y^{-1} x y x y^{-1} x y^{-1} x y^{-1} x y^{2} v_{0}^{-1} & & \text { since }\left(x y x y^{-1}\right)^{3}=1 \\
& =y x y^{2} x y^{-1} x y^{2} x y^{-2} v_{0}^{-1} & & \text { since }(x y)^{5}=1 \\
& =v_{4} v_{1} v_{0}^{-1} . & &
\end{aligned}
$$

Similarly $x^{-1} v_{2} x=v_{3}^{-1} v_{2} v_{4}$ and $x^{-1} v_{4} x=v_{4}^{-1} v_{2}^{-1} v_{1}^{-1} v_{4}^{-1}$ (with either one of these obtainable from other using the identity $v_{0} v_{2} v_{4} v_{1} v_{3}=1$ ), and it follows that the subgroup $K$ is normalized also by $x$ and is therefore normal in $G$. But furthermore the relations give:

$$
\begin{aligned}
v_{0}^{-1} v_{2}^{-1} v_{0} v_{2} & =y^{-2} x y^{-2} x y^{-2} x y x y^{-2} x y^{-2} x y^{2} x y^{2} x y^{-1} x y^{2} x y^{-1} \\
& =y^{-2} x y^{-2} x y^{-1} x y^{-1} x y x y^{-1} x y^{-1} x y^{-2} x y^{2} x y x y x y^{-1} x y x y x y^{-1}
\end{aligned}
$$




$$
\begin{aligned}
& =y^{-2} x y^{-1} x y x y^{-2} x y x y^{-1} x y x y^{-1} x y^{2} x y^{-1} x y^{-2} \\
& =y^{-2} x y^{-1} x y x y^{-1}\left(y^{-1} x y x\right)^{3} x y x y^{-1} x y^{-2} \\
& =y^{-2}\left(x y^{-1} x y\right)^{3} y^{2} \\
& =1
\end{aligned}
$$

conjugation by powers of $y$ then shows that $v_{i} v_{i+2}=v_{i+2} v_{i}$ for all $i$ (modulo 5), so $K$ is Abelian.

The factor group $G / K$ has presentation $\langle x, y, t| x^{2}=y^{5}=(x y)^{5}=t^{2}=(x t)^{2}=$ $\left.(y t)^{2}=(x y t)^{6}=\left(x y^{2}\right)^{3}=1\right)$, and by coset enumeration using CAYLEY [1] or GAP [5] this is easily shown to have order 120, being a direct product $A_{5} \times C_{2}$.

Finally, as in proof of Theorem 1, we show the group $G$ has a concrete representation of the required form by exhibiting a transitive permutation representation on the integers:

$$
\begin{aligned}
& x(5 k)=5 k \quad y(5 k)=5 k+1 \quad t(5 k)=5-5 k \\
& x(5 k+1)=5 k+13 \quad y(5 k+1)=5 k+2 \quad t(5 k+1)=9-5 k \\
& x(5 k+2)=5 k+14 \quad y(5 k+2)=5 k+3 \quad t(5 k+2)=8-5 k \\
& x(5 k+3)=5 k-9 \quad y(5 k+3)=5 k+4 \quad t(5 k+3)=7-5 k \\
& x(5 k+4)=5 k-8 \quad y(5 k+4)=5 k \quad t(5 k+4)=6-5 k \text {. }
\end{aligned}
$$

Again these permutations (defined for $k \in \mathbb{Z}$ ) satisfy the required relations and generate a transitive but imprimitive group on $\mathbb{Z}$, with residue classes modulo 5 this time as blocks of imprimitivity. Also the permutations induced by $v_{0}=\left(x y^{2}\right)^{3}$ and its conjugates generate a free Abelian group of rank 4, and the rest follows.

COROLLARY 2. For every positive integer $n$, there exists a regular map of type $\{5,5\}_{6}$ having $12 n^{4}$ vertices, $30 n^{4}$ edges and $12 n^{4}$ faces, on an orientable surface of genus $3 n^{4}+1$.

PROOF. Take the permutations given at the end of the above proof, modulo $10 n$; these generate a quotient of $G^{5,5,6}$ of order $120 n^{4}$, and the rest follows from material presented in the Introduction.

THEOREM 3. $G^{5,6,6}$ is an infinite insoluble group, with infinitely many finite quotients.

ProOF. Let $x, y$ and $t$ be generators for $G^{5,6,6}$ satisfying the relations $x^{2}=y^{5}=$ $(x y)^{6}=t^{2}=(x t)^{2}=(y t)^{2}=(x y t)^{6}=1$. As $(x y t)^{2}=x y t x y t=x y x t y t=$ $x y x y^{-1}$, we note the relation $\left(x y x y^{-1}\right)^{3}=1$ is satisfied also; in fact, by the Reidemeister-Schreier process (with transversal $\{1, t\}$ ), the index 2 subgroup generated by $x$ and $y$ has presentation $\left.\langle x, y| x^{2}=y^{5}=(x y)^{6}=\left(x y x y^{-1}\right)^{3}=1\right)$. 
Now let $K$ be the normal subgroup generated by $u=\left(x y x y x y^{-1} x y^{-1}\right)^{2}$ and its conjugates. By coset enumeration (using CAYLEY [1] or GAP [5]), the subgroup $K$ can be shown to have index 1440 in $\langle x, y, t\rangle$, or index 720 in $\langle x, y\rangle$, with corresponding quotients isomorphic to $S_{6} \times C_{2}$ and $S_{6}$ respectively. In particular, as the alternating group $A_{6}$ is simple, this shows $G^{5,6.6}$ is insoluble.

Next by the Abelianised form of the Reidemeister-Schreier process (for example, using the 'abelian quotient invariants' command in CAYLEY [1]), we find the Abelianisation $K / K^{\prime}$ of $K$ is isomorphic to $C_{3} \times \mathbb{Z}^{9}$ (the direct product of $C_{3}$ with a free Abelian group of rank 9). It follows that $G^{5,6,6}$ is infinite, and also that it has infinitely many (insoluble) quotients: for every positive integer $n$, a quotient of order $1440 n^{9}$ is obtainable by factoring out the pre-image of the normal subgroup of $K / K^{\prime}$ generated by the $C_{3}$ part and the $n$th powers of the generators of the free Abelian part of $K / K^{\prime}$.

COROLLARY 3. For every positive integer $n$, there exists a regular map of type $\{5,6\}_{6}$ having $120 n^{9}$ vertices, $360 n^{9}$ edges and $144 n^{9}$ faces, on an orientable surface of genus $48 n^{9}+1$.

This follows immediately from the proof of Theorem 3. As in the corollaries to Theorems 1 and 2, explicit generating permutations may be found for the automorphism groups of these maps, however they are too cumbersome to be given here. Also we remark that $G^{5,6,6}$ has many finite quotients different from those mentioned above, such as $S_{45}$ and $S_{46}$ for example. In particular, it is very unlikely that $G^{5,6,6}$ is Abelian-by-finite; see Section 4 for further discussion.

THEOREM 4. $G^{6,6,6}$ is an infinite insoluble group, with infinitely many finite quotients.

PROOF. This is an immediate consequence of the main result of [2]. Alternatively:

Let $x, y$ and $t$ be generators for $G^{6,6,6}$ satisfying the relations $x^{2}=y^{6}=(x y)^{6}=$ $t^{2}=(x t)^{2}=(y t)^{2}=(x y t)^{6}=1$. As in the proof of Theorem 3, the index 2 subgroup generated by $x$ and $y$ has presentation $\left\langle x, y \mid x^{2}=y^{6}=(x y)^{6}=\left(x y x y^{-1}\right)^{3}=1\right\rangle$.

This time let $K$ be the normal subgroup generated by $u=\left(x y^{2}\right)^{2}$ and its conjugates. By coset enumeration (using CAYLEY [1] or GAP [5]), the subgroup $K$ can be shown to have index 72 in $\langle x, y, t\rangle$, or index 36 in $\langle x, y\rangle$, with corresponding quotients isomorphic to $S_{3} \times S_{3} \times C_{2}$ and $S_{3} \times S_{3}$.

Next, as in the proof of Theorem 3, the Abelianised form of the ReidemeisterSchreier process may be used to find the Abelianisation $K / K^{\prime}$ of $K$; in fact $K / K^{\prime}$ is isomorphic to $C_{3} \times \mathbb{Z}^{4}$ (the direct product of $C_{3}$ with a free Abelian group of rank 4). It follows that $G^{6,6,6}$ is infinite, and also that it has infinitely many (soluble) quotients: for every positive integer $n$, a quotient of order $72 n^{4}$ is obtainable by factoring out the pre-image of the normal subgroup of $K / K^{\prime}$ generated by the $C_{3}$ part and the $n$th powers of the generators of the free Abelian part of $K / K^{\prime}$. 
Finally we note that $G^{6,6,6}$ has some insoluble quotients, such as $S_{5}, S_{14}$ and $A_{23}$; for example, there is a homomorphism from $G^{6,6,6}$ onto $S_{5}$ taking $x$ to $(1,2)(3,4), y$ to $(1,3)(2,4,5)$, and $t$ to $(2,3)(4,5)$. In particular, $G^{6,6,6}$ itself is insoluble.

COROLLARY 4. For every positive integer $n$, there exists a regular map of type $\{6,6\}_{6}$ having $6 n^{4}$ vertices, $18 n^{4}$ edges and $6 n^{4}$ faces, on an orientable surface of genus $3 n^{4}+1$. Also for all but finitely many $n$, there exists a regular map of type $\{6,6\}_{6}$ having $n ! / 12$ vertices, $n ! / 4$ edges and $n ! / 12$ faces, on an orientable surface of genus $n ! / 24+1$.

The first assertion here follows immediately from the proof above, and again explicit generating permutations may be found for the automorphism groups of these maps, however they are too cumbersome to be given here. The second assertion relies on further detail from [2], viz. that for all but finitely many $n$ there is a homomorphism from $G^{6,6,6}$ onto $S_{n}$ mapping the index 2 subgroup generated by $x$ and $y$ onto $A_{n}$.

\section{Final remarks}

A few obvious questions remain unanswered.

(1) Exactly for which triples $(p, q, r)$ is the group $G^{p, q, r}$ finite? This is the problem considered by Coxeter, and has still not been settled.

(2) Are any other of the groups $G^{p, q, r}$ Abelian-by-finite? or nilpotent-by-finite?

(3) At the other extreme: which of the groups $G^{p, q, r}$ have all but finitely many $A_{n}$ and $S_{n}$ as quotients? or (just) infinitely many $A_{n}$ and/or $S_{n}$ as quotients?

(4) In particular, what else can be said about the structure of the group $G^{5,6,6}$ ? Is it Abelian-by-finite? or does it have all but finitely many alternating and/or symmetric groups as quotients? It certainly has some $S_{n}$ as quotients, such as $S_{6}, S_{45}$ and $S_{46}$, but (so far) it has resisted all attempts by the author to prove it has infinitely many.

Of course one can play this game with $G^{p, q, r}$ for larger values of $p, q$ and $r$. It is not hard to show, for instance, that $G^{4,5,10}$ is infinite but soluble, with a rank 5 free Abelian normal subgroup of index 320 ; on the other hand, each of $G^{4,5,36}, G^{4,6,16}, G^{5,5,12}$ and $G^{5,6,14}$ has all but finitely many alternating and symmetric groups as quotients. Further details on these examples are available from the author on request.

\section{Acknowledgment}

The author would like to thank the Auckland University Research Committee for its support. 


\section{References}

[1] J. J. Cannon, 'An introduction to the group theory language CAYLEY', in: Computational group theory (ed. M. Atkinson) (Academic Press, San Diego, 1984) pp. 145-183.

[2] M. D. E. Conder, 'On the group $G^{6,6.6}$, Quart. J. Math. Oxford Ser. (2) 39 (1988), 175-183.

[3] H. S. M. Coxeter, 'The abstract groups $G^{m, n, p}$ ', Trans. Amer. Math. Soc. 45 (1939), 73-150.

[4] H. S. M. Coxeter and W. O. J. Moser, Generators and relations for discrete groups, 4th edition (Springer, Berlin, 1980).

[5] M. Schönert et al., GAP: Groups, algorithms and programming, Lehrstuhl D für Mathematik, RWTH Aachen (Aachen, 1992).

[6] S. E. Wilson, 'Cantankerous maps and rotary embeddings of $K_{n}$ ', J. Combin. Theory Ser. B 47 (1989), 262-273.

Department of Mathematics and Statistics

University of Auckland

Private Bag 92019

Auckland, New Zealand 